\title{
Phonon Contribution in Thermodynamics and Transport Properties of Ultrathin Ceramic Films
}

\author{
S.K. JaĆImovski ${ }^{a, *}$, J.P. ŠetrajČIĆ ${ }^{b}$, M.S. JaćImovski ${ }^{c}$ And V.V. Stojanović ${ }^{d}$ \\ ${ }^{a}$ Academy of Criminology and Police Studies, Zemun, Cara Dušana 196, Belgrade, Serbia \\ ${ }^{b}$ University of Novi Sad, Faculty of Sciences, Department of Physics, Novi Sad \\ Trg Dositeja Obradovića 4, Vojvodina, Serbia \\ ${ }^{c}$ University of Novi Sad, Faculty of Engineering, Novi Sad, Trg Dositeja Obradovića 6, Serbia \\ ${ }^{d}$ High Chemical Medical School, Vršac, Sterijina 113, Serbia
}

(Received March 26, 2014)

The dispersion law, density states of phonons, thermodynamics properties and thermal conductivity was analyzed in this paper. It has been shown that at low temperatures, thermal conductivity of thin film is considerably lower that of bulk-structure. It turned out that phonons in thin film require activation energy for exciting. This leads to extremely low specific heat and specific conductivity at low temperatures. Consequences of quoted facts were discussed in detail and their influence on kinetic and thermodynamic properties of thin films is estimated.

DOI: 10.12693 /APhysPolA.126.811

PACS: 73.50.-h, 73.61.-r, 74.25.fc, 66.30.Xj, 65.40.-b

\section{Introduction}

The scope of our study in this paper is limited to the analysis of the phonons, i.e. phonon behavior in thin layered structures or crystalline films, which implies the existence of two boundary surfaces perpendicular to a preferred direction. Besides that, these film-structures could be doped by foreign atoms from one or both sides of the boundary surfaces in which way the internal configuration of the atom distribution is disturbed.

Since elastic constants and atomic masses define phonon spectra and states, we conclude that they must be different in the film-structures with respect to the corresponding ones in the ideal unbounded and translational invariant crystalline structures. The change of mass distribution along one direction and the existence of the finite structure width along that direction introduces additional boundary conditions into the analysis of the phonon behavior.

We shall study the thin film "cut-off" from the ideal tetragonal crystalline structure with lattice constants $a_{x}=a_{y}=a$ and $a_{z}=3 a$. This structure has a finite width in the $z$-direction, while $X Y$-planes are assumed to be infinite, meaning that the structure possesses two infinite boundary surfaces (parallel to the unbounded $X Y$ -planes) lying at $z=0$ and $z=L$ (Fig. 1). The number of the atoms located along $z$-direction is assumed to be $N_{z}$, and it is also assumed that torsion constants $\mathcal{C}_{\alpha \beta}(\alpha \neq \beta)$ can be neglected with respect to the elongation constants $\mathcal{C}_{\alpha \alpha}[1]$.

We have decided to study phonon behavior in the above mentioned film-structures for two reasons. Phonons are the basic elementary excitations in the condensed matter which have the decisive role in the

* corresponding author; e-mail: jacimovskis@gmail.com

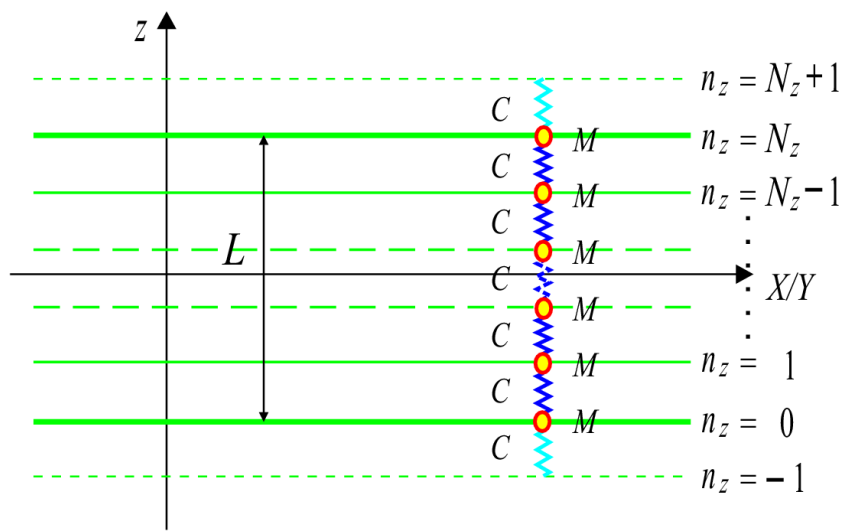

Fig. 1. Sight of crystalline film-structure model.

creation of the Cooper pairs of electrons in the low-temperature superconductivity. On the other hand, although the existence of phonons and Cooper pairs in the high-temperature superconductive ceramics is experimentally established [2], the very mechanism of the superfluid charge transfer is not yet resolved $[1,3]$. Taking into account the technical and technological treatments for the production of these high-temperature superconductors - they are small-grain crystalline structures (of small dimensions with pronounced boundaries) produced by doping, more precisely sputtering by guest atoms in certain stoichiometric ratio $[4,5]$, it is necessary to formulate the corresponding theoretical model.

The simplest model for the bounded structures is the ideal crystalline film. Within the framework of this model we shall study only and exclusively the influence of the system boundaries onto spectra and states of phonons and their contribution to the basic physical properties of the system. In order to consider also the influence of the doping, we shall study the spectra and states of phonons in the deformed films and estimate what has the 
stronger influence to the change of the system behavior: the existence of the boundary surfaces or the disturbance of the internal distribution and type of atoms inside the system.

The starting point of our study will be the standard Hamiltonian of the phonon system $[6,7]$ in the nearest neighbors' approximation

$$
H_{I D}=\frac{1}{2} \sum_{\boldsymbol{n}} \frac{p_{\boldsymbol{n}}^{2}}{M_{\boldsymbol{n}}}+\frac{1}{4} \sum_{\boldsymbol{n}, \boldsymbol{\lambda}} \mathcal{C}_{\boldsymbol{n}, \boldsymbol{\lambda}}\left(\boldsymbol{u}_{\boldsymbol{n}}-\boldsymbol{u}_{\boldsymbol{n}+\boldsymbol{\lambda}}\right)^{2},
$$

where $\boldsymbol{p}_{\boldsymbol{n}}$ and $\boldsymbol{u}_{\boldsymbol{n}}$ are the momentum and displacement of the atom of mass $M_{\boldsymbol{n}}$ at the crystal site $\boldsymbol{n}=a\left(n_{x} \boldsymbol{e}_{x}+\right.$ $\left.n_{y} \boldsymbol{e}_{y}+3 n_{z} \boldsymbol{e}_{z}\right)$, while $\mathcal{C}_{\boldsymbol{n}, \boldsymbol{\lambda}} \equiv \mathcal{C}_{\boldsymbol{\lambda}, \boldsymbol{n}}$ is Hooke's elastic constants between the atom at the site $\boldsymbol{n}$ and its neighboring atoms at the site $\boldsymbol{m}=\boldsymbol{n}+\boldsymbol{\lambda}, \boldsymbol{\lambda}=a\left(\boldsymbol{e}_{x}+\boldsymbol{e}_{y}+3 \boldsymbol{e}_{z}\right)$.

One of the most important aims is to study if the minimal frequencies of the atoms in the film are non-vanishing, i.e. does the phonon energy spectrum possess the gap. In the structures where such gap exists, there can arise the damping or the elimination of the acoustical phonons [1,4] so that there exist only the phonons of optical type. This would result in the film behavior like a "frozen" structure until certain corresponding activation temperature is reached - the temperature necessary for the creation of the phonons in the film [8], since below that temperature, real (acoustical) phonons cannot be present ${ }^{\dagger}$.

Taking this into account, the presence of the phonon gap might represent the possible explanation of the fact [9] that thin films represent better ordered thermodynamical systems and that they have higher critical superconductive temperature than the corresponding bulkstructures. Since the change in the properties of the anisotropic structures, is caused by the change in the dispersion law, it is necessary to study the behavior of relevant physical quantities in order to obtain a more complete picture about these processes.

\section{Dispersion law and states of phonons in films}

In Sect. 2 we derive the dispersion law for phonons and calculate the possible phonon states in the above mentioned crystalline films.

The Hamiltonian of the phonon subsystem of the model film-structure in the nearest neighbors approximation [10] is given in the form of expression (1.1), where

$$
\begin{aligned}
& -\frac{N_{\beta}}{2} \leq n_{\beta} \leq \frac{N_{\beta}}{2}, \quad N_{\beta} \approx 10^{8}, \quad \beta \in(x, y), \\
& 0 \leq n_{z} \leq N_{z}, \quad N_{z}=\frac{L}{3 a} \approx 20,
\end{aligned}
$$

so that it can be written in the expanded form as

$$
H_{\mathrm{FS}}=\frac{1}{2} \sum_{\alpha} \sum_{n_{x}, n_{y}} \sum_{n_{z}=0}^{N_{z}} \frac{\left(p_{n_{x}, n_{y}, n_{z}}^{\alpha}\right)^{2}}{M_{n_{x}, n_{y}, n_{z}}}
$$

†For example, electrons would move in such a structure without friction up these temperatures, i.e. they would behave like ideal conductors.

$$
\begin{aligned}
& +\frac{1}{4} \sum_{\alpha} \sum_{n_{x}, n_{y}}\left\{\mathcal{C}_{-1}^{\alpha}\left(u_{n_{x}, n_{y}, 0}^{\alpha}\right)^{2}+\mathcal{C}_{0}^{\alpha}\left[\left(u_{n_{x}+1, n_{y}, 0}^{\alpha}\right.\right.\right. \\
& \left.-u_{n_{x}, n_{y}, 0}^{\alpha}\right)^{2}+\left(u_{n_{x}-1, n_{y}, 0}^{\alpha}-u_{n_{x}, n_{y}, 0}^{\alpha}\right)^{2} \\
& +\left(u_{n_{x}, n_{y}+1,1}^{\alpha}-u_{n_{x}, n_{y}, 0}^{\alpha}\right)^{2}+\left(u_{n_{x}, n_{y}-1,0}^{\alpha}-u_{n_{x}, n_{y}, 0}^{\alpha}\right)^{2} \\
& \left.+\left(u_{n_{x}, n_{y}, 1}^{\alpha}-u_{n_{x}, n_{y}, 0}^{\alpha}\right)^{2}+\left(u_{n_{x}, n_{y}, 0}^{\alpha}\right)^{2}\right] \\
& +\mathcal{C}_{1}^{\alpha}\left[\left(u_{n_{x}, n_{y}, 1}^{\alpha}-u_{n_{x}, n_{y}, 2}^{\alpha}\right)^{2}+\left(u_{n_{x}, n_{y}, 1}^{\alpha}-u_{n_{x}, n_{y}, 0}^{\alpha}\right)^{2}\right] \\
& +\sum_{n_{z}=1}^{N_{z}-1} \mathcal{C}_{n_{z}}^{\alpha}\left[\left(u_{n_{x}+1, n_{y}, n_{z}}^{\alpha}-u_{n_{x}, n_{y}, n_{z}}^{\alpha}\right)^{2}\right. \\
& +\left(u_{n_{x}-1, n_{y}, n_{z}}^{\alpha}-u_{n_{x}, n_{y}, n_{z}}^{\alpha}\right)^{2}+\left(u_{n_{x}, n_{y}+1, n_{z}}^{\alpha}\right. \\
& \left.\left.-u_{n_{x}, n_{y}, n_{z}}^{\alpha}\right)^{2}+\left(u_{n_{x}, n_{y}-1, n_{z}}^{\alpha}-u_{n_{x}, n_{y}, n_{z}}^{\alpha}\right)^{2}\right] \\
& +\sum_{n_{z}=2}^{N_{z}-2} \mathcal{C}_{n_{z}}^{\alpha}\left[\left(u_{n_{x}, n_{y}, n_{z}+1}^{\alpha}-u_{n_{x}, n_{y}, n_{z}}^{\alpha}\right)^{2}\right. \\
& \left.+\left(u_{n_{x}, n_{y}, n_{z}-1}^{\alpha}-u_{n_{x}, n_{y}, n_{z}}^{\alpha}\right)^{2}\right] \\
& +\mathcal{C}_{N_{z}-1}^{\alpha}\left[\left(u_{n_{x}, n_{y}, N_{z}-1}^{\alpha}-u_{n_{x}, n_{y}, N_{z}}^{\alpha}\right)^{2}\right. \\
& \left.+\left(u_{n_{x}, n_{y}, N_{z}-1}^{\alpha}-u_{n_{x}, n_{y}, N_{z}-2}^{\alpha}\right)^{2}\right] \\
& +\mathcal{C}_{N_{z}}^{\alpha}\left[\left(u_{n_{x}+1, n_{y}, N_{z}}^{\alpha}-u_{n_{x}, n_{y}, N_{z}}^{\alpha}\right)^{2}+\left(u_{n_{x}-1, n_{y}, N_{z}}^{\alpha}\right.\right. \\
& \left.-u_{n_{x}, n_{y}, N_{z}}^{\alpha}\right)^{2}+\left(u_{n_{x}, n_{y}+1, N_{z}}^{\alpha}-u_{n_{x}, n_{y}, N_{z}}^{\alpha}\right)^{2} \\
& +\left(u_{n_{x}, n_{y}-1, N_{z}}^{\alpha}-u_{n_{x}, n_{y}, N_{z}}^{\alpha}\right)^{2}+\left(u_{n_{x}, n_{y}, N_{z}-1}^{\alpha}\right. \\
& \left.\left.-u_{n_{x}, n_{y}, N_{z}}^{\alpha}\right)^{2}+\left(u_{n_{x}, n_{y}, N_{z}}^{\alpha}\right)^{2}\right] \\
& \left.+\mathcal{C}_{N_{z}+1}^{\alpha}\left(u_{n_{x}, n_{y}, N_{z}}^{\alpha}\right)^{2}\right\} \text {. }
\end{aligned}
$$

This Hamiltonian describes the film-structure model presented in Fig. 1. It enables further theoretical analysis of the properties, specific effects and changes in the phonon behavior in above mentioned translational non-invariant systems. All changes and specific effects which can occur in the system, will be treated as a strict consequence of the contribution of the mechanical vibrations of the atoms of the crystal lattice under the influence of the presence of boundary surfaces and asymmetric distribution of the atoms along one preferred $(z)$ crystallographic direction in that system.

\section{Ideal film-structures}

The concept of the ideal film means here the model of the crystal bounded by two parallel surfaces which can "breathe" (no rigid walls) along one crystallographic direction (which we choose for the positive direction of $z$-axis) perpendicular to the boundary surfaces and unbounded in the two other remaining directions. Further- 
more, besides boundaries, there are no other defects in the ideal film, so inside the boundaries we encounter single atom tetragonal structure.

The Hamiltonian, expression (2.1), adapted to the above mentioned model can be separated into two parts: the first one $H_{\mathrm{S}}$, which includes "surface" terms and the second one $H_{\mathrm{B}}$, which includes "bulk" terms subject to the conditions (see Fig. 1):

$$
\begin{aligned}
& M_{n_{x}, n_{y}, n_{z}} \equiv M, \\
& \mathcal{C}_{n_{x} n_{y} n_{z} ; n_{x} \pm 1, n_{y} n_{z}}^{\alpha}=\mathcal{C}_{n_{x} n_{y} n_{z} ; n_{x} n_{y} \pm 1, n_{z}}^{\alpha} \\
& \quad=\mathcal{C}_{n_{x} n_{y} n_{z} ; n_{x} n_{y} n_{z} \pm 1}^{\alpha} \equiv \mathcal{C}_{\alpha} .
\end{aligned}
$$

Since there are no layers for $n_{z} \leq-1$ and for $n_{z} \geq N_{z}+1$, we must include the following condition, too:

$$
\left.u_{n_{x}, n_{y}, l}^{\alpha}=0, \quad l \leq-1 \wedge l \geq N_{z}+1 \quad \text { (i.e. } l \notin\left[0, N_{z}\right]\right) .
$$

If we would assign $\mathcal{C}_{-1}^{\alpha}=\mathcal{C}_{N_{z}+1}^{\alpha}=0$, then the boundary atoms (for $n_{z}=0$ and $n_{z}=N_{z}$ ) would be "frozen", i.e. we would have the effects of rigid walls [11]. In this way, the expression for the total Hamiltonian of the ideal crystalline film obtains the following form:

$$
H_{\mathrm{IF}}=H_{\mathrm{S}}+H_{\mathrm{B}},
$$

where

$$
\begin{aligned}
& H_{\mathrm{S}}=\frac{1}{2 M} \sum_{\alpha} \sum_{n_{x}, n_{y}}\left[\left(p_{n_{x}, n_{y}, 0}^{\alpha}\right)^{2}+\left(p_{n_{x}, n_{y}, N_{z}}^{\alpha}\right)^{2}\right] \\
& +\frac{1}{4} \sum_{\alpha} \mathcal{C}_{\alpha} \sum_{n_{x}, n_{y}}\left[2\left(u_{n_{x}, n_{y}, 0}^{\alpha}\right)^{2}+2\left(u_{n_{x}, n_{y}, N_{z}}^{\alpha}\right)^{2}\right. \\
& +\left(u_{n_{x}, n_{y}, N_{z}-1}^{\alpha}-u_{n_{x}, n_{y}, N_{z}}^{\alpha}\right)^{2} \\
& +\left(u_{n_{x}, n_{y}, 1}^{\alpha}-u_{n_{x}, n_{y}, 0}^{\alpha}\right)^{2} \\
& +\left(u_{n_{x}, n_{y}, 0}^{\alpha}-u_{n_{x}+1, n_{y}, 0}^{\alpha}\right)^{2} \\
& +\left(u_{n_{x}, n_{y}, 0}^{\alpha}-u_{n_{x}-1, n_{y}, 0}^{\alpha}\right)^{2} \\
& +\left(u_{n_{x}, n_{y}, 0}^{\alpha}-u_{n_{x}, n_{y}+1,0}^{\alpha}\right)^{2} \\
& +\left(u_{n_{x}, n_{y}, 0}^{\alpha}-u_{n_{x}, n_{y}-1,0}^{\alpha}\right)^{2} \\
& +\left(u_{n_{x}, n_{y}, N_{z}}^{\alpha}-u_{n_{x}+1, n_{y}, N_{z}}^{\alpha}\right)^{2} \\
& +\left(u_{n_{x}, n_{y}, N_{z}}^{\alpha}-u_{n_{x}-1, n_{y}, N_{z}}^{\alpha}\right)^{2} \\
& +\left(u_{n_{x}, n_{y}, N_{z}}^{\alpha}-u_{n_{x}, n_{y}+1, N_{z}}^{\alpha}\right)^{2} \\
& \left.+\left(u_{n_{x}, n_{y}, N_{z}}^{\alpha}-u_{n_{x}, n_{y}-1, N_{z}}^{\alpha}\right)^{2}\right] \text {, } \\
& H_{\mathrm{B}}=\frac{1}{2 M} \sum_{\alpha} \sum_{n_{x}, n_{y}}\left(p_{n_{x}, n_{y}, n_{z}}^{\alpha}\right)^{2}+\frac{1}{4} \sum_{\alpha} \mathcal{C}_{\alpha} \\
& \times \sum_{n_{x}, n_{y}}\left\{\sum _ { n _ { z } = 1 } ^ { N _ { z } - 1 } \left[\left(u_{n_{x}+1, n_{y}, n_{z}}^{\alpha}-u_{n_{x}, n_{y}, n_{z}}^{\alpha}\right)^{2}\right.\right.
\end{aligned}
$$

$$
\begin{aligned}
& +\left(u_{n_{x}-1, n_{y}, n_{z}}^{\alpha}-u_{n_{x}, n_{y}, n_{z}}^{\alpha}\right)^{2} \\
& +\left(u_{n_{x}, n_{y}+1, n_{z}}^{\alpha}-u_{n_{x}, n_{y}, n_{z}}^{\alpha}\right)^{2} \\
& \left.+\left(u_{n_{x}, n_{y}-1, n_{z}}^{\alpha}-u_{n_{x}, n_{y}, n_{z}}^{\alpha}\right)^{2}\right] \\
& +\sum_{n_{z}=2}^{N_{z}-2}\left[\left(u_{n_{x}, n_{y}, n_{z}+1}^{\alpha}-u_{n_{x}, n_{y}, n_{z}}^{\alpha}\right)^{2}\right. \\
& \left.+\left(u_{n_{x}, n_{y}, n_{z}-1}^{\alpha}-u_{n_{x}, n_{y}, n_{z}}^{\alpha}\right)^{2}\right] \\
& +\left(u_{n_{x}, n_{y}, N_{z}-1}^{\alpha}-u_{n_{x}, n_{y}, N_{z}-2}^{\alpha}\right)^{2} \\
& +\left(u_{n_{x}, n_{y}, 1}^{\alpha}-u_{n_{x}, n_{y}, 2}^{\alpha}\right)^{2}
\end{aligned}
$$

Energy spectra and states will be determined by using Green's function method. For that purpose we observe two/time commutator Green's function [6, 7] with adequate equation of motion

$$
\begin{aligned}
& G_{\boldsymbol{n}, \boldsymbol{m}}^{\alpha}\left(t-t^{\prime}\right) \equiv\left\langle\left\langle u_{\alpha ; \boldsymbol{n}}(t) \mid u_{\alpha ; \boldsymbol{m}}\left(t^{\prime}\right)\right\rangle\right\rangle \\
& \quad=\Theta\left(t-t^{\prime}\right)\left\langle\left[u_{\alpha ; \boldsymbol{n}}(t), u_{\alpha ; \boldsymbol{m}}\left(t^{\prime}\right)\right]\right\rangle_{0}, \\
& M \frac{\mathrm{d}^{2}}{\mathrm{~d} t^{2}} G_{\boldsymbol{n}, \boldsymbol{m}}^{\alpha}\left(t-t^{\prime}\right)=-\mathrm{i} \hbar \delta_{\boldsymbol{n}, \boldsymbol{m}} \delta\left(t-t^{\prime}\right) \\
& \quad+\frac{\Theta\left(t-t^{\prime}\right)}{\mathrm{i} \hbar}\left\langle\left[\left[p_{\alpha ; \boldsymbol{n}}(t), H(t)\right], u_{\alpha ; \boldsymbol{m}}\left(t^{\prime}\right)\right]\right\rangle_{0} .
\end{aligned}
$$

Calculating corresponding commutators and using the partial spatial Fourier-transformation (because the translational symmetry has been disturbed only along $z$-direction)

$$
\begin{aligned}
& G_{n_{x}, n_{y}, n_{z} ; m_{x}, m_{y}, m_{z}}^{\alpha}(\omega)=\frac{1}{N} \sum_{k_{x}, k_{y}} G_{n_{z}, m_{z}}^{\alpha}\left(k_{x}, k_{y} ; \omega\right) \\
& \quad \times \mathrm{e}^{-\mathrm{i} a\left[\left(n_{x}-m_{x}\right) k_{x}+\left(n_{y}-m_{y}\right) k_{y}\right]},
\end{aligned}
$$

we obtain the system of $N_{z}^{I}+1$ homogeneous algebraic-difference equations [12]:

$$
G_{n_{z}-1, m_{z}}^{\alpha}+\varrho_{k}^{\alpha} G_{n_{z}, m_{z}}^{\alpha}=\mathcal{K} \delta_{n_{z}, m_{z}},
$$

where $G_{n_{z}, m_{z}}^{\alpha} \equiv G_{n_{z} m_{z}}^{\alpha}\left(k_{x}, k_{y} ; \omega\right), \mathcal{K}=\frac{\mathrm{i} \hbar}{2 \pi C_{\alpha}}, k=$ $\sqrt{k_{x}^{2}+k_{y}^{2}}$, while the determinant of that system of equations is

$$
\mathcal{D}_{N_{z}^{I}+1}(\varrho)=\left|\begin{array}{ccccccccc}
\varrho-\varepsilon & 1 & 0 & 0 & \ldots & 0 & 0 & 0 & 0 \\
1 & \varrho & 1 & 0 & \ldots & 0 & 0 & 0 & 0 \\
0 & 1 & \varrho & 1 & \ldots & 0 & 0 & 0 & 0 \\
& & & & & & & \\
\vdots & \vdots & \vdots & \vdots & \ddots & \vdots & \vdots & \vdots & \vdots \\
0 & 0 & 0 & 0 & \ldots & 1 & \varrho & 1 & 0 \\
0 & 0 & 0 & 0 & \ldots & 0 & 1 & \varrho & 1 \\
0 & 0 & 0 & 0 & \ldots & 0 & 0 & 1 & \varrho-\gamma
\end{array}\right|_{N_{z}+1}
$$

where 


$$
\begin{aligned}
& \varrho_{k}^{\alpha}=\frac{\omega^{2}}{\Omega_{\alpha}^{2}}-4 \sin ^{2} \frac{a k_{x}}{2}-4 \sin ^{2} \frac{a k_{y}}{2}-2 \equiv \varrho, \\
& \Omega_{\alpha}=\sqrt{\frac{C_{\alpha}}{M}} .
\end{aligned}
$$

In order to find the spectra of the allowed phonon energies amounts, we must determine the zeroes of the determinant (2.8) i.e. to solve the equality

$$
\begin{gathered}
\mathcal{D}_{N_{z}+1}(\varrho ; \varepsilon, \gamma) \equiv 0 \Longrightarrow \varrho=\varrho_{\mu}(\varepsilon, \gamma), \\
\mu=1,2,3, \ldots, N_{z}^{I}+1 .
\end{gathered}
$$

This task in general is not analytically solvable (it can be solved numerically with the given parameters $\varepsilon, \gamma$ and $N_{z}^{I}$ ). When $\varepsilon=\gamma=0$ (model of the lost surfaces [13]), we obtain analytical solution

$$
\mathcal{D}_{N_{z}^{I}+1}(\varrho)=\varrho \mathcal{P}_{N_{z}^{I}}(\varrho)-\mathcal{P}_{N_{z}^{I}-1}(\varrho) \equiv \mathcal{P}_{N_{z}^{I}+1}(\varrho),
$$

where the determinant (2.8) of the system of Eq. (2.7) is expressed directly through the characteristic Chebyshev polynomials of order $N_{z}^{I}$. In this case, we obtain an expression that gives us the phonon dispersion law in ultrathin and structurally non-deformed crystalline nanofilm

$$
\begin{aligned}
& { }^{I} E_{k_{x} k_{y}}^{\alpha}(\mu) \equiv \hbar^{I} \omega_{k_{x} k_{y}}^{\alpha}(\mu)=E_{I}^{\alpha} \sqrt{\mathcal{G}_{\mu}^{I}+\mathcal{F}_{k_{x} k_{y}}} \\
& \quad=2 \hbar \Omega_{\alpha} \sqrt{\mathcal{G}_{\mu}^{I}+\mathcal{F}_{k_{x} k_{y}}},
\end{aligned}
$$

where

$$
\mathcal{G}_{\mu}^{I} \equiv \sin ^{2} \frac{a k_{z}(\mu)}{2}, \quad \mathcal{F}_{k_{x} k_{y}} \equiv \sin ^{2} \frac{a_{x} k_{x}}{2}+\sin ^{2} \frac{a_{y} k_{y}}{2},
$$

where $k_{x}$ and $k_{y}$ are virtually continual within the interval $[0, \pi / a]$, and $k_{z}$ has distinctly discrete values

$$
k_{z}(\mu)=\frac{\pi}{a} \frac{\mu}{N_{z}^{I}+2}, \quad \mu=1,2,3, \ldots, N_{z}^{I}+1 .
$$

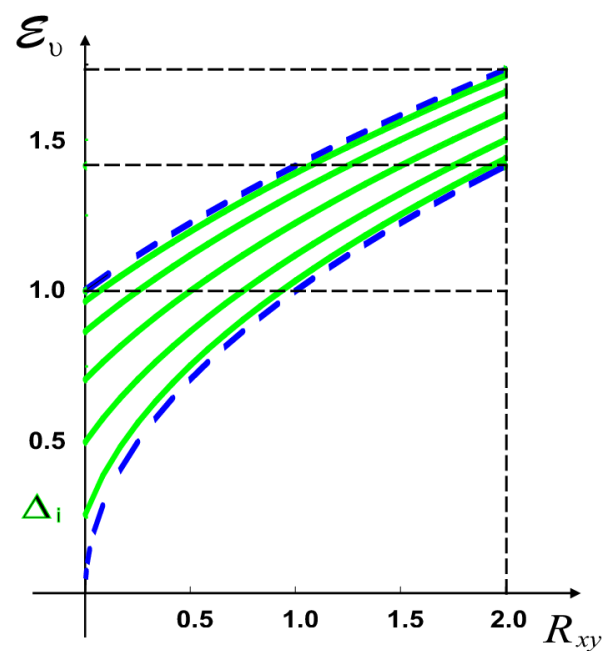

Fig. 2. Phonon spectra in the ideal ultrathin crystalline films.

We represent graphically this energy spectrum in Fig. 2 vs. $X Y$-plane vector $k^{2}=k_{x}^{2}+k_{y}^{2}$ :

$$
{ }^{I} \mathcal{E}_{\mu}^{z} \equiv\left({ }^{I} E_{k_{x} k_{y}}^{z}(\mu) / E_{I}^{z}\right)^{2}={ }^{I} \mathcal{E}_{k_{z}(\mu)}^{z}\left(\mathcal{F}_{k_{x} k_{y}}\right)
$$

Figure 2 represents energy spectrum of phonons in the ideal (ultrathin $N_{z}=4$ ) crystalline films vs. two-dimensional ( $X Y$ planar) wave vector. Within the band of bulk energies with continual spectrum (bulk limits are denoted by solid dashed lines) one can notice five allowed discrete phonon energies in the film studied (thin solid lines). One can notice the narrowing of the energy band and the existence of the energy gap.

One can clearly see from the plot explicate discreteness of the allowed energy levels of phonons in the ideal film with respect to the continuum of these values for the corresponding bulk-structures. All three acoustic frequencies in bulk-structures vanish when three-dimensional (spatial) vector $k=|\boldsymbol{k}|$ vanishes, while the minimal frequencies of phonons in the thin ideal film-structure are

$$
\begin{aligned}
\Delta_{i} & \equiv\left(\omega_{\alpha}^{I}\right)_{\min } \equiv \omega_{\alpha}^{I}\left(k_{x}=k_{y}=0, k_{z}=k_{z}^{\min }\right) \\
& \approx \Omega_{\alpha} \frac{\pi}{N_{z}^{I}}>0 .
\end{aligned}
$$

On the other hand, maximal values of the frequencies of acoustic branches in the ideal infinite crystal tend to the value $\left(\omega_{\alpha}^{\mathrm{B}}\right)_{\max }=2 \Omega_{\alpha} \sqrt{3}$ when $k_{\alpha} \rightarrow \pi / a, \alpha=x, y, z$, while in the studied ideal film they are

$$
\begin{gathered}
\left(\omega_{\alpha}^{I}\right)_{\max } \equiv \omega_{\alpha}^{I}\left(k_{x}=k_{y}=\frac{\pi}{a}, k_{z}=k_{z}^{\max }\right) \\
\approx 2 \Omega_{\alpha} \sqrt{3}\left[1-\frac{\pi^{2} / 12}{\left(N_{z}^{I}\right)^{2}}\right]<\left(\omega_{\alpha}^{\mathrm{B}}\right)_{\max } .
\end{gathered}
$$

It can be also seen from the same figure that the width of the energy band in the film is narrower. From expressions (2.14) and (2.15) we can determine the total narrowing of the band of allowed energies of the phonons in the film-structures with respect to the bulk band

$$
\begin{aligned}
\mathcal{W}_{\alpha}^{I} & \equiv \hbar\left\{\left(\omega_{\alpha}^{\mathrm{B}}\right)_{\max }-\left[\left(\omega_{\alpha}^{I}\right)_{\max }-\left(\omega_{\alpha}^{I}\right)_{\min }\right]\right\} \\
& \approx \hbar \Omega_{\alpha} \frac{\pi\left(N_{z}^{I}+1\right)}{\left(N_{z}^{I}\right)^{2}}>0 .
\end{aligned}
$$

\section{Phonon thermodynamics of thin film-structures}

Forasmuch as the properties of anisotropic structures are conditioned by the change of dispersion law, it is necessary to observe behavior of certain thermodynamic properties towards obtainment of better understanding about those properties. Phonon participation in thermodynamic properties (or heat capacitance temperature behavior, i.e. generally - in heat transferring) in thin film was found in our previous paper [14-18].

Getting that, when $k \rightarrow 0$ (in long-wave approximation: $\left.4\left[\sin ^{2}\left(a k_{x} / 2\right)+\sin ^{2}\left(a k_{y} / 2\right)\right] \approx a^{2} k^{2}, k^{2}=k_{x}^{2}+k_{y}^{2}\right)$, energies of all three phonon branches have non-zero values, there can be utilized dispersion relations (2.12), in somewhat simplified form

$$
E(\boldsymbol{k})=\sqrt{a^{2} k^{2} E_{0}^{2}+\Delta_{f}^{2}},
$$

where

$$
\Delta_{f}=a k_{z}^{\min } E_{0}, \quad E_{0} \equiv \hbar \sqrt{\frac{C_{\alpha}}{M}} .
$$

It should be specifically emphasized that verification of phonon dispersion law at very low values of $k$ is virtually 
impossible, so that verification of existence of phonon gap detects itself in measurement of low temperature thermal capacitances in film and corresponding ideal structure.

The thermal capacitance is analyzed, whereby at first internal energy is calculated in terms of standard form $[6,7,11,19]$ :

$$
U_{f}=3 \sum_{k_{x}, k_{y}, k_{z}} E(\boldsymbol{k})\left(\mathrm{e}^{E(\boldsymbol{k}) / \theta}-1\right)^{-1} .
$$

Going over from sum in last expression to integral in accordance with the formula ${ }^{\ddagger}$ :

$$
\begin{aligned}
& \sum_{k_{x}, k_{y}, k_{z}} \longrightarrow 3\left(N_{z}+1\right) \sum_{k_{x}, k_{y}} \longrightarrow \frac{3 N_{x} N_{y}\left(N_{z}+1\right) a^{2}}{4 \pi^{2}} \\
& \quad \times \int_{0}^{2 \pi} \mathrm{d} \varphi \int_{0}^{k_{\max }} k \mathrm{~d} k,
\end{aligned}
$$

and taking $k_{\max } \approx k_{\mathrm{D}}=\sqrt[3]{6 \pi^{2}}$, after suitable notations

$$
\eta \equiv \sqrt{\frac{N_{z}^{2}}{3}+N_{z}+1}, \quad \zeta \equiv \sqrt{1+\left(\frac{N_{z}+2}{\pi} \sqrt[3]{6 \pi^{2}}\right)^{2}}
$$

and adequate operations, expression for internal energy has been obtained in form

$$
\begin{aligned}
& U_{f}(x)=\frac{3 N_{f}}{4 \pi^{2}} \frac{\Delta_{f}^{4}}{E_{0}^{3}} x^{2}\left\{\left[Z_{2}\left(\frac{1}{x}\right)-\eta^{2} Z_{2}\left(\frac{\eta}{x}\right)\right.\right. \\
& \left.+\eta^{2} \zeta^{2} Z_{2}\left(\frac{\eta \zeta}{x}\right)-\zeta^{2} Z_{2}\left(\frac{\zeta}{x}\right)\right]+4 x\left[Z_{3}\left(\frac{1}{x}\right)\right. \\
& \left.-\eta Z_{3}\left(\frac{\eta}{x}\right)+\eta \zeta Z_{3}\left(\frac{\eta \zeta}{x}\right)-\zeta Z_{3}\left(\frac{\zeta}{x}\right)\right] \\
& +6 x^{2}\left[Z_{4}\left(\frac{1}{x}\right)-Z_{4}\left(\frac{\eta}{x}\right)+Z_{4}\left(\frac{\eta \zeta}{x}\right)\right. \\
& \left.\left.-Z_{4}\left(\frac{\zeta}{x}\right)\right]\right\},
\end{aligned}
$$

where the symbol $x$ is introduced for reduced temperature: $x=\frac{\theta}{\Delta_{f}}, N_{f}=N_{x} N_{y}\left(N_{z}+1\right)$ and $Z_{r}(X)=$ $\sum_{j=1}^{\infty} j^{-r} \mathrm{e}^{-j X}$ - the functions are called Dyson's functions.

For finding of expression for the thermal capacitance per a unit cell (here: per an atom), the standard definitional form $[6,7,11,19]$ is used:

$$
C_{f}=\frac{1}{N_{f}} \frac{\partial U_{f}}{\partial T} \equiv \frac{k_{\mathrm{B}}}{N_{f}} \frac{\partial U_{f}}{\partial \theta}=\frac{1}{\Delta_{f}} \frac{k_{\mathrm{B}}}{N_{f}} \frac{\partial U_{f}}{\partial x} .
$$

‡The transition $\sum_{\boldsymbol{k}} \rightarrow \int \mathrm{d} \boldsymbol{k}=\int \mathrm{d}^{3} k$ of Descartes coordinates for film must be carried out to cylindrical coordinates due to finite thickness.

$$
\begin{aligned}
& \text { In accordance with that it is obtained } \\
& \begin{array}{l}
C_{f}(x)=\frac{3 k_{\mathrm{B}}}{4 \pi^{2}}\left(\frac{\Delta_{f}}{E_{0}}\right)^{3}\left\{\left[Z_{1}\left(\frac{1}{x}\right)-\eta^{3} Z_{1}\left(\frac{\eta}{x}\right)\right.\right. \\
\left.\quad+\eta^{3} \zeta^{3} Z_{1}\left(\frac{\eta \zeta}{x}\right)-\zeta^{3} Z_{1}\left(\frac{\zeta}{x}\right)\right]+6 x\left[Z_{2}\left(\frac{1}{x}\right)\right. \\
\left.\quad-\eta^{2} Z_{2}\left(\frac{\eta}{x}\right)+\eta^{2} \zeta^{2} Z_{2}\left(\frac{\eta \zeta}{x}\right)-\zeta^{2} Z_{2}\left(\frac{\zeta}{x}\right)\right] \\
+18 x^{2}\left[Z_{3}\left(\frac{1}{x}\right)-\eta Z_{3}\left(\frac{\eta}{x}\right)+\eta \zeta Z_{3}\left(\frac{\eta \zeta}{x}\right)\right. \\
\left.\quad-\zeta Z_{3}\left(\frac{\zeta}{x}\right)\right]+24 x^{3}\left[Z_{4}\left(\frac{1}{x}\right)-Z_{4}\left(\frac{\eta}{x}\right)\right. \\
\left.\left.+Z_{4}\left(\frac{\eta \zeta}{x}\right)-Z_{4}\left(\frac{\zeta}{x}\right)\right]\right\} .
\end{array}
\end{aligned}
$$

It is known that the phonon part in thermal capacitance of the system is described with cubic temperature dependence. By introducing nondimensional reduced temperature, this dependence amounts to: $C_{b}(x)=$ $\frac{12}{5} \pi^{4} N_{b} k_{\mathrm{B}}\left(\frac{\Delta_{f}}{E_{\mathrm{D}}}\right)^{3} x^{3}$. For comparison of these dependencies, these and expression (3.6) are divided by the constant: $C_{0}=\frac{k_{\mathrm{B}}}{2}\left(\frac{\Delta_{f}}{E_{\mathrm{D}}}\right)^{3}$, whose dimension is equal to dimension of thermal capacitance, and nondimensional properties are compared: $\mathcal{C}_{f / b} \equiv \frac{C_{f / b}}{C_{0}}$. In Fig. 3 there are shown relative (nondimensional) thermal capacitances of bulk $(b)$ and film-structure $(f)$ subject to the relative temperature $x$ in low (a) and very low temperature region.
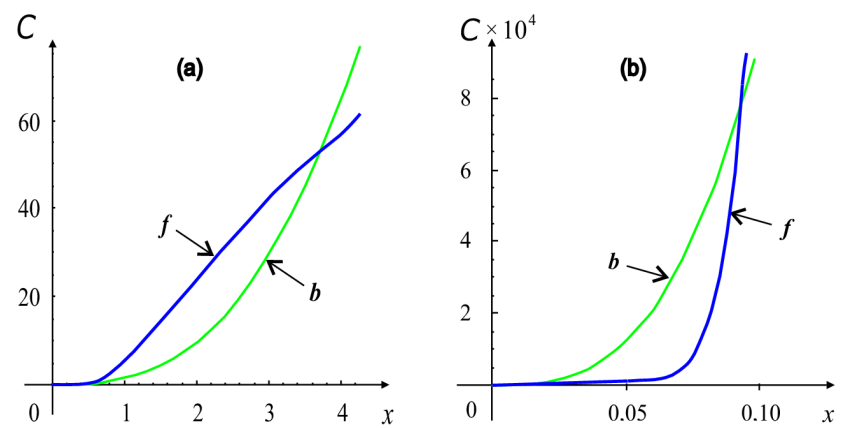

Fig. 3. Thermal capacitance vs. temperature at low and extremely low temperatures.

In Fig. 4 there are shown relative (nondimensional) thermal capacitances of bulk $(b)$ and film-structure $(f)$ samples versus relative temperature $x$, for ultrathin $N_{z}=3$ (a), thin $N_{z}=8$ (b), and thick $N_{z}=48$ (c) film-structures, in comparison with bulk ones.

It can be seen that in low-temperature region (Fig. 3) thermal capacitance of film is lower than that of massive specimens, whereas at the intermediate temperatures situation is reversed [12]. Intersect point of two curves at low temperatures is moving — with increase of film thick- 

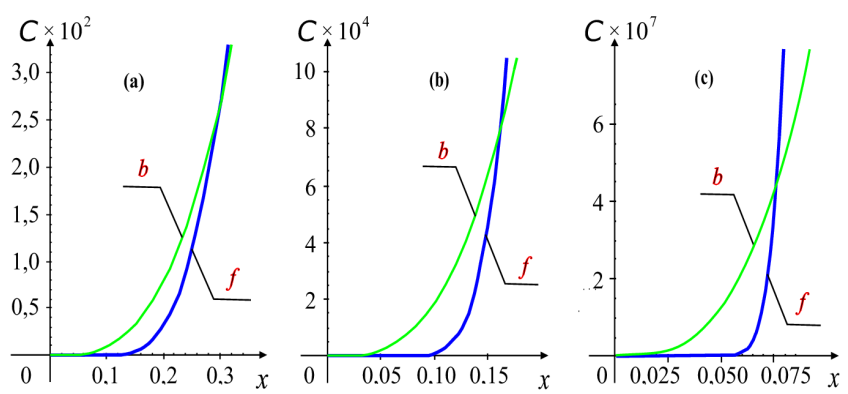

Fig. 4. Thermal capacitance in low temperature regime for ultrathin, thin, thick films.

ness - towards lower temperatures (a)-(c). Besides, it is noticeable that thermal capacitance of film with decrease of temperature declines faster than that of corresponding ideal structure, or slowly rises with the increase of temperature - to a certain upper temperature. Hence, for film heating from certain lower to a certain upper temperature, it is necessary to use more thermal energy per mass unit than for heating the same quantity of corresponding (with the identical crystallographical parameters) unbounded structure to the same temperature. It is in accordance with the fact that phonons in film have non-zero excitation energy.

\section{Thermal conductivity of thin film crystalline}

Using the formula

$$
\kappa=D C \varrho_{M},
$$

where $\kappa$ is the coefficient of the thermal conductivity, $D$ - the diffusion coefficient, $C$ - the specific heat and $\varrho_{M}$ - the density, we shall determine the coefficient thermal conductivity of thin film. The analysis of the coefficient is the great practical interest, since it determines the heat isolation and a number of other properties of these structures [20].

The diffusion coefficient $D$ (strictly speaking the diffusion tensor $D_{i j}$ ) will be found via the use of Kubo's formula [21]. The temperature dependence of the film density will be determined using the Green function method.

All calculations will be carried out for ideal boundary conditions in films, and for specific boundary conditions on its surfaces, which can be chosen in a way which leads to some useful effects. The optimal choice of boundary conditions is the practical goal of investigations pertaining to deformed structures.

Starting from the Kubo formula [22], one obtains the following expression defining the diffusion coefficient:

$$
D_{i j}^{f}(k)=\lim _{\delta \rightarrow 0+} \int_{0}^{\infty} \mathrm{d} t \mathrm{e}^{-\delta t}\left\langle\hat{v}_{i}(0) \hat{v}_{j}(t)\right\rangle,
$$

where $\hat{v}_{i}$ and $\hat{v}_{j}$ are the velocity operators in the Heisenberg representation, and $\delta$ is the perturbation parameter and indexes $i, j$ takes values $x, y, z$.

The average is taken over great canonical ensemble, i.e.

$$
\langle\ldots\rangle=\operatorname{Sp}\left\{\mathrm{e}^{(\Phi+\mu N-H) / \theta}(\ldots)\right\},
$$

where $\Phi$ is thermodynamical potential, $\mu$ - chemical potential, and $H$ - the Hamiltonian of the system and $\theta=k_{\mathrm{B}} T$.

Using procedure given in [12] we obtain correlation function in the form of

$$
\left\langle v_{f}(t) v_{f}(0)\right\rangle=\frac{\hbar C_{\alpha}}{M^{2} \omega_{k}}\left(\frac{\mathrm{e}^{-\mathrm{i} \omega_{k} t}}{\mathrm{e}^{\hbar \omega_{k} / \theta}-1}-\frac{\mathrm{e}^{\mathrm{i} \omega_{k} t}}{\mathrm{e}^{-\hbar \omega_{k} / \theta}-1}\right) .
$$

In accordance with the general formula (4.2) and expression (2.3) the diffusion coefficient is given by

$$
\begin{aligned}
D_{f} & \equiv D_{i j}^{f}(k)=\mid \lim _{\delta \rightarrow 0+} \frac{\hbar C_{\alpha}}{M^{2} \omega_{k}} \int_{0}^{\infty} \mathrm{e}^{-\delta t}\left(\frac{\mathrm{e}^{-\mathrm{i} \omega_{k} t}}{\mathrm{e}^{\hbar \omega_{k} / \theta}-1}\right. \\
- & \left.\frac{\mathrm{e}^{\mathrm{i} \omega_{k} t}}{\mathrm{e}^{-\hbar \omega_{k} / \theta}-1}\right) \mathrm{d} t \mid=\frac{\hbar C_{\alpha}}{M^{2}\left\langle\omega_{k}^{2}\right\rangle},
\end{aligned}
$$

where

$$
\begin{aligned}
\omega_{\boldsymbol{k}}^{\alpha} & \equiv \omega_{k_{x}, k_{y}, k_{z}}^{\alpha} \\
& =2 \Omega^{\alpha} \sqrt{\sin ^{2} \frac{a k_{x}}{2}+\sin ^{2} \frac{a k_{y}}{2}+\sin ^{2} \frac{a k_{z}}{2}} .
\end{aligned}
$$

The notation used in (4.5) is

$$
\begin{aligned}
& \Omega^{\alpha} \equiv \sqrt{\frac{C_{\alpha}}{M}}, \quad k_{x}=\frac{\pi \mu_{x}}{N_{x}^{I} a}, \quad k_{y}=\frac{\pi \mu_{y}}{N_{y}^{I} a}, \\
& \mu_{x / y} \in\left[-\frac{N_{x / y}^{I}}{2},+\frac{N_{x / y}^{I}}{2}\right], \quad N_{x / y}^{I} \approx 10^{8},
\end{aligned}
$$

while $k_{z}$ are solutions of the transcendental equation

$$
\begin{aligned}
& \cot \left(N_{z}-1\right) a k_{z} \\
& =\frac{4 \cos ^{3} a k_{z}-2\left(\rho_{0}+\rho_{N}\right) \cos ^{2} a k_{z}+\left(\rho_{0} \rho_{N}-3\right) \cos a k_{z}+\rho_{0} \rho_{N}}{-\sin a k_{z}\left[4 \cos ^{2} a k_{z}+2\left(\rho_{0}+\rho_{N}\right) \cos a k_{z}+\left(1-\rho_{0} \rho_{N}\right)\right]},
\end{aligned}
$$

where

$$
\rho=\frac{M \omega^{2}}{C_{\alpha}}-4\left(\sin ^{2} \frac{a k_{x}}{2}+\sin ^{2} \frac{a k_{y}}{2}\right)-2 .
$$

In the "cut-off" case $[1,23]$ (the approximation $\rho_{0}=$ $\rho_{N}=0$ will be called that) we obtain

$$
k_{z}=\frac{\pi \mu_{z}}{a\left(N_{z}^{I}+2\right)}, \quad \mu_{z}=1,2,3, \ldots, N_{z}^{I}+1 .
$$

It can be seen that levels $k_{x}$ and $k_{y}$ are equidistant, while, due to the structure deformations, levels $k_{z}$ have lost equidistant properties.

To determine the density, we first calculate the density correction caused by molecular vibrations. Starting with the standard expression [14]:

$$
\begin{aligned}
\boldsymbol{u}_{\boldsymbol{n}} & =\sum_{j, \boldsymbol{k}} \sqrt{\frac{\hbar}{2 M N \omega_{\boldsymbol{k}}^{j}}}\left[b_{j}^{+}(\boldsymbol{k}) \mathrm{e}^{-\mathrm{i}\left(\boldsymbol{k} \boldsymbol{n}-\omega_{\boldsymbol{k}}^{j} t\right)}\right. \\
& \left.+b_{j}(\boldsymbol{k}) \mathrm{e}^{\mathrm{i}\left(\boldsymbol{k} \boldsymbol{n}-\omega_{\boldsymbol{k}}^{j} t\right)}\right] \boldsymbol{e}_{j}(\boldsymbol{k}), \quad j \in(x, y, z),
\end{aligned}
$$

for molecular displacements, we can find the averages of square displacements 


$$
\begin{aligned}
& \sum_{\boldsymbol{n}}\left\langle u_{\boldsymbol{n}}^{2}\right\rangle \equiv N_{f}\left\langle u^{2}\right\rangle=\sum_{j, \boldsymbol{k}} \frac{\hbar}{2 M \omega_{\boldsymbol{k}}^{j}}\left[1+2\left\langle\hat{n}_{\boldsymbol{k}}\right\rangle\right], \\
& \left\langle\hat{n}_{\boldsymbol{k}}\right\rangle=\frac{1}{\mathrm{e}^{E_{\boldsymbol{k}} / \theta}-1} .
\end{aligned}
$$

Introduction of the notation $\left\langle u_{0}^{2}\right\rangle=\frac{1}{N} \sum_{\boldsymbol{k}} \frac{\hbar}{2 M\left\langle\omega_{\boldsymbol{k}}\right\rangle}$, one can find in (4.10):

$$
\begin{gathered}
\left\langle u^{2}\right\rangle-\left\langle u_{0}^{2}\right\rangle=\frac{3}{2 \pi} \frac{\hbar}{M \omega_{\mathrm{D}}} \frac{\Delta_{f}^{2}}{E_{0}^{2}}\left\{Z_{1}\left(\frac{1}{x}\right)-\eta Z_{1}\left(\eta \frac{1}{x}\right)\right. \\
\left.+\frac{1}{x}\left[Z_{2}\left(\frac{1}{x}\right)-\eta Z_{2}\left(\eta \frac{1}{x}\right)\right]\right\} .
\end{gathered}
$$

The density of the film is given by the formula

$$
\begin{aligned}
\rho_{M} & =\frac{M}{\left\langle\left(a_{0}+u\right)^{3}\right\rangle}=\frac{M}{\left\langle a_{0}^{3}\right\rangle} \frac{1}{1+3 \frac{\left\langle u^{2}\right\rangle}{\left\langle a_{0}^{2}\right\rangle}} \rho_{M} \\
& \approx \rho_{0}^{\mathrm{M}}\left(1-\frac{3\left\langle u^{2}\right\rangle}{\left\langle a_{0}^{2}\right\rangle}\right) .
\end{aligned}
$$

Using Debye's approximation [14] we shall substitute $\left\langle u^{2}\right\rangle$ in (4.12) with $\left\langle u^{2}\right\rangle-\left\langle u_{0}^{2}\right\rangle$ from formula (4.11). Thus, we obtain

$$
\begin{aligned}
\rho_{M} & =\rho_{0}^{M}\left(1-\frac{3}{2 \pi} \frac{\hbar^{2} \Delta_{f}^{2} x}{M\left\langle a_{0}\right\rangle^{2} E_{\mathrm{D}} E_{0}^{2}}\left\{Z_{1}\left(\frac{1}{x}\right)\right.\right. \\
& \left.\left.-\eta Z_{1}\left(\eta \frac{1}{x}\right)+\frac{1}{x}\left[Z_{2}\left(\frac{1}{x}\right)-\eta Z_{2}\left(\eta \frac{1}{x}\right)\right]\right\}\right) .
\end{aligned}
$$

Substituting (4.4), (3.6) and (4.13) in (4.1) we finally obtain the expression for the thermal conductivity coefficient of thin film-structures

$$
\begin{aligned}
\kappa_{f} & =\frac{3 k_{\mathrm{B}}}{2 \pi} \frac{\hbar \Omega^{\alpha 2}}{M\left\langle\omega_{k}^{2}\right\rangle}\left(\frac{\Delta_{f}}{E_{0}}\right)^{2}\left\{\frac { 1 } { x } \left[\left(\mathrm{e}^{1 / x}-1\right)^{-1}\right.\right. \\
& \left.-\eta^{3}\left(\mathrm{e}^{\eta / x}-1\right)^{-1}\right]+3\left[Z_{1}\left(\frac{1}{x}\right)-\eta^{2} Z_{1}\left(\eta \frac{1}{x}\right)\right] \\
& +6 x\left[Z_{2}\left(\frac{1}{x}\right)-\eta Z_{2}\left(\eta \frac{1}{x}\right)\right]+6 x^{2}\left[Z_{3}\left(\frac{1}{x}\right)\right. \\
& \left.\left.-Z_{3}\left(\eta \frac{1}{x}\right)\right]\right\} \rho_{0}^{M}\left(1-\frac{3}{2 \pi} \frac{\hbar^{2}}{M\left\langle a_{0}\right\rangle^{2} E_{\mathrm{D}}}\left(\frac{\Delta_{f}}{E_{0}^{2}}\right)^{2}\right. \\
& \times x\left\{Z_{1}\left(\frac{1}{x}\right)-\eta Z_{1}\left(\eta \frac{1}{x}\right)+x\left[Z_{2}\left(\frac{1}{x}\right)\right.\right. \\
& \left.\left.\left.-\eta Z_{2}\left(\eta \frac{1}{x}\right)\right]\right\}\right) .
\end{aligned}
$$

Formula (4.14) was analyzed numerically as a function of the scaled temperature $x=\theta / \Delta_{f}$. The notation

$$
\lambda_{b / f} \equiv \frac{\kappa_{b / f}(x)}{\kappa_{0}}, \quad \kappa_{0}=\frac{8}{9}\left(6 \pi^{2}\right)^{-2 / 3} \frac{\hbar}{a^{3}}\left(\frac{\Delta_{f}}{E_{0}}\right)^{3},
$$

representing scaled thermal conductivity, is introduced into formula (4.14). The dependence of scaled thermal conductivity on the scaled temperature is given in Fig. 5: (a) at extremely low temperature (up to $5 \mathrm{~K}$ ) - left, (b) at higher temperature (in the range $T>5 \mathrm{~K}$ ) - right.
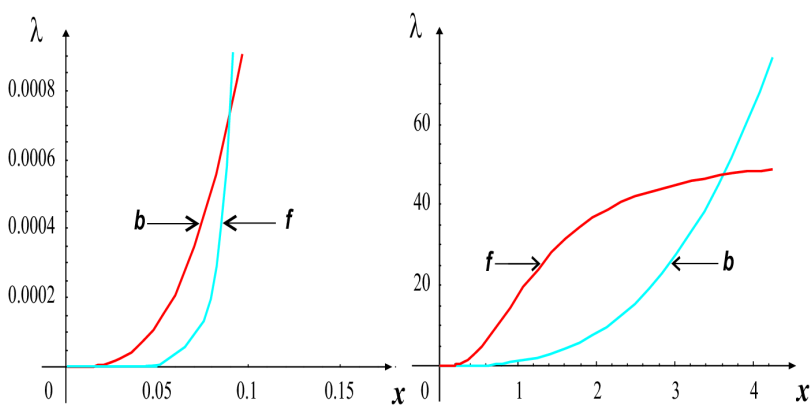

Fig. 5. Thermal conductivity versus temperature.

It can be seen from figures quoted that, at extremely low temperatures $T \leq 4 \mathrm{~K}$ and at temperatures $T \geq$ $180 \mathrm{~K}$, the thermal conductivity of a film is lower than that of a bulk. In the temperature range $4<T<180 \mathrm{~K}$ the thermal conductivity of a bulk is lower than that of a film.

At the end of this section we would like to point out an important fact: since Eq. (4.6) does not have solution $k_{z}=0$, all phonon energies in film have a gap. Consequently, to excite the phonons in films some activation energy is necessary.

\section{Conclusion}

Studying and comparing the phonon spectra and states in the ideal unbounded and nondeformed (bulk) structures and the structures with broken translational symmetry (films) we have reached the following conclusions.

1. Mechanical vibrations in bulk-structures are plane waves in all directions, while in the films they represent the superposition of the standing waves in $z$-directions (perpendicular to the boundary surfaces) and plane waves in $X Y$-planes (parallel to boundary surfaces).

2. The amplitude of phonon displacements in the films depends on the film width and it is $\sim 10^{4} \sqrt{2 / N_{z}}$ times higher than in the ideal structures. This indicates their larger elastic "maneuvering space" without any negative effect to the mechanical properties of the given material (for example no breaking of interatomic bonds) which leads to higher resistance and higher melting point of the films with respect to bulk samples.

3. All three acoustic frequencies in bulk-structures vanish for $\boldsymbol{k} \rightarrow 0$, while in the films they tend toward some minimal value depending on the film width. This means that phonons in the films possess the energy gap, that for their excitation (creation) one should spend certain energy, i.e. heat 
them up to certain activation temperature, meaning that the system up to that temperature behaves as the "frozen" one, as if the phonons were not present.

4. Phonon gap, besides depending on the film width, depends also on the type of the atoms and their distribution along $z$-direction and also on the stoichiometric relation of the atoms injected in the films.

5. The densities of phonon states and Debye's frequencies have lower values in the films than in the corresponding bulk-structures. This implies that in the films, phonon excitations are more "difficult" to appear, that they are less "present" and that created acoustic phonons of the optical type (above the activation temperature) are energetically "softer" than the classical ones which appear in the bulkstructures.

6. Since phonons with Debye's frequencies define thermal and electrical properties of the materials, this means that films are worse thermal and electrical conductors.

These analyses show that the films are better superconductors than the corresponding bulk samples, made from the same material with the same crystalline structure. This statement, which is an experimental fact is supported by the following of our results.

1. In the films there appear standing phonon waves along $z$-directions, the collective property specific for the macroscopic quantum-mechanical state which is the characteristics of the superconductors. In the ideal structures, where there exist only plane phonon waves, there is no such property.

2. The appearance of the energy gap in the phonon spectrum of the films means that up to the activation temperature these systems behave as completely frozen, i.e. without any mechanical vibrations which would cause the real resistance to the electrical current conduction.

3. Lower values of Debye's frequencies in the films could result in the higher values of (BCS) matrix elements of the effective electron-electron interaction. The attraction between paired electrons is stronger, and one must spend more energy for their destruction, so the critical temperature of these systems is higher.

Since phonons with Debye's frequencies are responsible for electrical and thermal induced transport properties of material $[6,7]$, it follows that the nanofilm structure will be inferior electrical and thermic conductor in contrast with the relative massive structures, providing there is no chemical and structural differences between them.

On the other hand, it is well known fact that the more inferior electrical conductor materials is (under normal conditions), the better superconductor it becomes
$[11,14]$. Due to that, the experimental fact can be concluded and justified, that in spatially very confined structures more qualitative superconductive properties have been achieved.

In the region of low temperatures, the thermal capacitance of film is lower than in massive structures, while in medium temperatures it is reversed. Intersect point of two curves at low temperatures is moving - with increase of film thickness - towards lower temperatures. Besides, it is noticeable that thermal capacitance of film with decrease of temperature declines faster than that of corresponding ideal structure, or slowly rises with the increase of temperature - to a certain upper temperature.

Hence, for film heating from certain lower to a certain upper temperature, it is necessary to use more thermal energy per mass unit than for heating the same quantity of corresponding (with the identical crystallographical parameters) unbounded structure to the same temperature.

It is well known that poorer electric conductors are better superconductors, so that in ultrathin films it is possible to achieve much better superconducting properties!

The results obtained show that the thermal conductivity coefficient of a film is considerably lower than that of a bulk at low temperature, where the thermal conductivity of bulk decreases as $T^{3}$. This result is practically applicable: sandwich of several films would be a better thermal insulator than the bulk-structure of the same thickness.

In accordance with the Wiedemann-Franz law, the electrical conductivity is proportional to thermal conductivity. This leads to conclusion that films are worse electrical conductors than bulk-structures of the same material.

It could be interesting to estimate the superconductive properties, since worse conductors are, in principle, better superconductors.

The results obtained here are compared to theoretical results as well as to the experimental data from Refs. [24-26].

\section{Acknowledgments}

The authors of this paper owe the greatest thanks their teacher, mentor and friend, unfortunately - a recently deceased Academician, Prof. Dr. Bratislav S. Tošić. His proper and quality oriented suggestions had crucial impact on the value and importance of this work.

This work was financially supported by the Serbian Ministry of Education, Science and Technological Development (projects ON-171039 and TR-34019), and by the Ministry of Sciences and Technology of the Republic of Srpska (project: 19/6-020/961-23/12) as well as by the Vojvodina Provincial Secretariat for Science and Technological Development (grant 114-451-2048). 


\section{References}

[1] B.S. Tošić, J.P. Šetrajčić, R.P. Djajić, D.Lj. Mirjanić, Phys. Rev. B 36, 9094 (1987).

[2] L.L. Chang, L. Esaki, Phys. Today 45, 36 (1992).

[3] J.G. Bednorz, K.A. Müller, Rev. Mod. Phys. 60, 585 (1988).

[4] J.P. Šetrajčić, R.P. Djajić, D.Lj. Mirjanić, B.S. Tošić, Phys. Scr. 42, 732 (1990).

[5] D.R. Harshman, A.P. Jr. Mills, Phys. Rev. B 45, 10684 (1992).

[6] G.D. Mahan, Many-Particle Physics, Plenum, London 1983.

[7] W. Jones, N.H. March, Theoretical Solid State Physics, Dover, New York 1985.

[8] R.P. Djajić, J.P. Šetrajčić, D.Lj. Mirjanić, B.S. Tošić, Int. J. Mod. Phys. B 1, 1001 (1987).

[9] CRC Handbook of Chemistry and Physics, 70th ed., Chemical Rubber, Cleveland 1989-1990.

[10] B.S. Tošić, D.Lj. Mirjanić, J.P. Šetrajčić, Spectra of Elementary Excitations in the Anisotropic FilmStructure, Physical Society of the Republic of Srpska, Banja Luka 1995 (in Serbian).

[11] A.A. Maradudin, in: Proc. 33th Winter School of Theoretical Physics, Karpacz (Poland), Lecture Notes in Physics 285, Ed. T. Paszkiewicz, Springer, Berlin 1987.

[12] D. Popov, S.K. Jaćimovski, B.S. Tošić, J.P. Šetrajčić, Physica A 317, 129 (2003).

[13] M.G. Cottam, D.R. Tilley, Introduction to Surface and Superlattice Excitations, University Press, Cambridge 1989.

[14] S. Lazarev, M.R. Pantić, S.M. Stojković, B.S. Tošić, J.P. Šetrajčić, J. Phys. Chem. Solids 61, 931 (2000).
[15] S.K. Jaćimovski, J.P. Šetrajčić, B.S. Tošić, V.D. Sajfert, Mater. Sci. Forum 453-454, 33 (2004).

[16] J.P. Šetrajčić, V.M. Zorić, S.M. Vučenović, D.Lj. Mirjanić, V.D. Sajfert, S.K. Jaćimovski, D.I. Ilić, Mater. Sci. Forum 555, 291 (2007).

[17] D.I. Ilić, S.M. Vučenović, S.K. Jaćimovski, V.M. Zorić, J.P. Šetrajčić, in: Low-Dimensional Materials Synthesis, Assembly, Property Scaling, and Modeling, Eds. M. Shim, M. Kuno, X.-M. Lin, R. Pachter, S. Kumar, Materials Research Society, San Francisco (USA) 2007, Vol. 50, p. 1.

[18] J.P. Šetrajčić, D.Lj. Mirjanić, S.M. Vučenović, D.I. Ilić, B. Markoski, S.K. Jaćimovski, V.D. Sajfert, V.M. Zorić, Acta Phys. Pol. A 115, 778 (2009).

[19] J. Callaway, Quantum Theory of the Solid State, Academic Press, New York 1991.

[20] P. Hyldgaard, G.D. Mahan, in: Thermal Conductivity, Vol. 23, Tehnomic, Lancaster 1996, p. 172.

[21] R.J. Kubo, Phys. Soc. Jpn 12, 570 (1957).

[22] V.M. Agranovich, M.D. Galanin, Migration of Electron Energy Excitations in Condensed Matter, Nauka, Moscow 1978 (in Russian).

[23] S.B. Lazarev, D.Lj. Mirjanić, M.R. Pantić, B.S. Tošić, J.P. Šetrajčić, J. Phys. Chem. Sol. 60, 846 (1999).

[24] A.J. Bullen, K.E. O'Hara, D.G. Cahill, O. Monteiro, A. von Keudell, J. Appl. Phys. 88, 6317 (2000).

[25] D.H. Santamore, M.C. Cross, Phys. Rev. B 63 , 184306 (2001).

[26] W.E. Bies, R. Radtke, H. Ehrenreich, J. Appl. Phys. 88, 1498 (2000). 